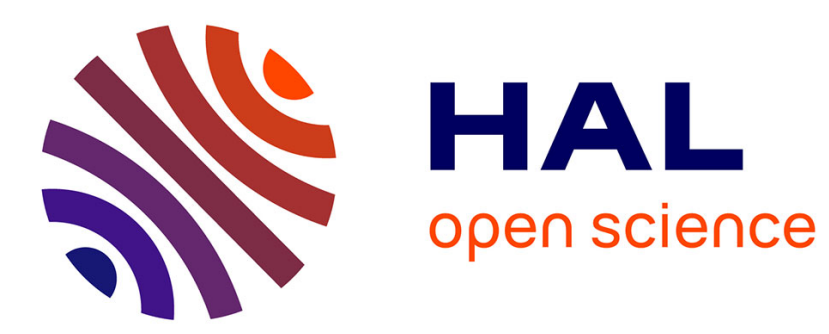

\title{
A Reduced Order representation of the Poincaré-Steklov operator: an application to coupled multi-physics problems
}

Matteo Aletti, Damiano Lombardi

\section{- To cite this version:}

Matteo Aletti, Damiano Lombardi. A Reduced Order representation of the Poincaré-Steklov operator: an application to coupled multi-physics problems. International Journal for Numerical Methods in Engineering, 2017. hal-01396286

\section{HAL Id: hal-01396286 \\ https://auf.hal.science/hal-01396286}

Submitted on 14 Nov 2016

HAL is a multi-disciplinary open access archive for the deposit and dissemination of scientific research documents, whether they are published or not. The documents may come from teaching and research institutions in France or abroad, or from public or private research centers.
L'archive ouverte pluridisciplinaire HAL, est destinée au dépôt et à la diffusion de documents scientifiques de niveau recherche, publiés ou non, émanant des établissements d'enseignement et de recherche français ou étrangers, des laboratoires publics ou privés. 


\title{
A Reduced Order representation of the Poincaré-Steklov operator: an application to coupled multi-physics problems
}

\author{
Matteo Aletti, Damiano Lombardi \\ Inria Paris \& Sorbonne Universités UPMC Univ Paris 6, France
}

\begin{abstract}
This work investigates a model reduction method applied to coupled multi-physics systems. The case in which a system of interest interacts with an external system is considered. An approximation of the Poincaré-Steklov operator is computed by simulating, in an offline phase, the external problem when the inputs are the LaplaceBeltrami eigenfunctions defined at the interface. In the online phase, only the reduced representation of the operator is needed to account for the influence of the external problem on the main system. An online basis enrichment is proposed in order to guarantee a precise reduced-order computation. Several test-cases are proposed on different fluid-structure couplings.
\end{abstract}

\section{Introduction}

The present work deals with the study of a Reduced-Order Method to approximate the solution of coupled multi-physics systems. In particular, we investigate the case in which one system of interest, described by a possibly non-linear Partial Differential Equation (PDE) interacts with one (or more) other systems through its boundaries. Numerous applications in science and engineering are characterized by different compartments in interaction, think for instance to thermal-fluid-structural or electro-mechanical-fluid couplings. In several cases, one is not interested in the solution to all the systems, but only in the solution of a "main" system, that will be denoted by $\mathcal{P}_{1}$. The objective is thus to be able to compute precisely the solution of this system (by using a full-order classical method) but to reduce the computational costs associated to the solution of the systems in interaction with it (denoted as $\mathcal{P}_{2}$ ). This results in a significant speed up of the problem simulation, in the case in which the size of $\mathcal{P}_{2}$ is larger than that of $\mathcal{P}_{1}$. Indeed, classically, there are two ways to deal with coupled systems: a monolithic approach in which all the systems are simultaneously solved, or a Domain Decomposition method (see [1] for a complete review of the method). In the latter a fixed point iteration is adopted, in which all the systems are separately solved and share the boundary data. In the case in which the 
secondary systems need a large number of degrees of freedom to be accurately solved, the computational cost associated to both the approaches may be large.

In this first work, for sake of simplicity, we made the assumption that there is only one system to be reduced and that it is described by linear PDEs. In this case the interaction of the linear system with $\mathcal{P}_{1}$ may be described by the Poincaré-Steklov operator. With a slight abuse of notation we call PoincaréSteklov operator the one associated to a generic linear PDE, even if historically this name refers to the case in which the secondary system is described by a Laplace equation (see [2] for a first analysis of the problem).

The need to set up efficient solvers and to decouple the solution of the problems in interaction is related to the ability to solve the problem at the interface. The use of the Poincaré-Steklov operator as a preconditioner in fluid-structure interaction iterations was investigated in [3]. An efficient non-linear coupling strategy was devised in [4] to set up an uncertainty quantification method applied to networks of coupled systems. Unfortunately, the problem at the interface is in general not sparse and ill-conditioned (see [1]). To tackle this issue several strategies were proposed in the literature. They can be broadly divided into two classes: "local" and spectral approximations. A local approximation of the Poincaré-Steklov operator consists of solving one or more external problems $\left(\mathcal{P}_{2}\right.$ in the present work) in a strip localised around the interface. Such a method was proposed for example in [5], for applications in hydrology. A similar procedure, based on a two-scale method, was presented in [6]: a local problem in a strip localised around the interface is solved and then, thanks to the residual, a global correction is computed. A different strategy consists of approximating the leading part of the action of the Poincaré-Steklov operator through a spectral decomposition. Such an approach was proposed in $[7,8]$ in the case of elliptic problems and a multiscale version was proposed in [9] for applications in heterogeneous media. An approximation of the PoincaréSteklov operator via a Padé expansion was detailed in [10] for the study of the vibrations in fluid-structure couplings. In [11] the Poincaré-Steklov operator is computed in the context of the wave propagation in elastodynamics by considering a family of smooth functions at the interface and by solving the problem $\mathcal{P}_{2}$ by taking these functions as inputs. In the recent work [12] a compressed sensing approach is proposed to retrieve the discretised Poincaré-Steklov operator for coupled Helmoltz problems. The method consists of probing, randomly, the matrix associated to the Poincaré-Steklov operator, by selecting inputs from a kernel space and performing a direct full-order simulation for a small number of them.

In the present work, a low rank decomposition of the Poincaré-Steklov operator is computed by a Reduced-Order Modeling method. In the literature, similar works were recently proposed, based on a Reduced-Basis framework $[13,14,15,16]$. In these, multi-domain systems are considered for coupled linear steady problems. The framework proposed in these works deals with parametrized systems whereas in the present work we focus on the acceleration of single-scenario simulations. In the present case, a parametrization can be considered for the problem $\mathcal{P}_{1}$; a remark on the possibility to extend the pro- 
posed approach to parametrized problems $\mathcal{P}_{2}$ is proposed at the end of Section 3.

The proposed approach is not applied to a specific set of problems but it is meant to be applied to a rather broad class of systems. As for most methods proposed in Reduced-Order Modeling ([17]), the strategy can be divided into two phases: an offline phase and an online one. In the offline phase, a deterministic sampling of the functional space of the input for the $\mathcal{P}_{2}$ at the interface is considered and the output is saved, at the interface. This is similar to what was proposed in [13], in which harmonic functions at the interface are used and the offline phase is somewhat independent from the coupling. The output of $\mathcal{P}_{2}$ at the interface is used to get a Low Rank Decomposition of the Poincaré-Steklov operator. In order to make the method more robust in cases in which the inputs coming from $\mathcal{P}_{1}$ are outside the space spanned by the sampled functions used to construct the database, an online update of the reduced Steklov representation is performed, in the spirit of the methods proposed in $[18,19]$.

The advantages of the proposed framework are the following: it is a straightforward method, allowing to speed up coupled multi-physics time dependent systems in a domain decomposition approach. Moreover, the offline phase is completely independent of the nature of $\mathcal{P}_{1}$ and of the coupling. Its main limitations concern the assumptions made on $\mathcal{P}_{2}$ : in the present work, $\mathcal{P}_{2}$, once discretized, has to be autonomous and linear. Although these assumptions are quite restrictive, they are fulfilled by a wide range of applications, for which a system of interest interacts with surrounding media or compartments whose dynamics is linear.

The structure of the work is as follows: in Section 2, the mathematical formulation of the problem is detailed and few properties of the Poincaré-Steklov operator are recalled. In Section 3 the numerical method is described and then, in Section 4, its numerical properties are investigated from a theoretical point of view. In the last section, some numerical experiments are proposed to validate the method.

\section{Problem formulation}

In this section, the mathematical formulation of the problem is detailed. The aim is to deal with systems of partial differential equations defined on different domains, in interaction. To simplify, in this work, we investigate the case in which two problems are coupled, namely $\mathcal{P}_{1}$ and $\mathcal{P}_{2}$. Let the coupled systems be defined on a time interval $[0, T]$ and a space domain $\Omega \subseteq \mathbb{R}^{d}$, an open subset, such that $\Omega=\Omega_{1} \cup \Omega_{2}$ where $\Omega_{1}$ is the domain on which $\mathcal{P}_{1}$ is defined and $\Omega_{2}$ is the domain on which $\mathcal{P}_{2}$ is defined. The interface between the two, where the coupling conditions are enforced, is denoted by $\Gamma:=\bar{\Omega}_{1} \cap \bar{\Omega}_{2}$. The problem of interest is $\mathcal{P}_{1}$, on which there are no hypotheses, it can be a generic non-linear PDE. The following working hypotheses on the problem $\mathcal{P}_{2}$ as well as on the coupling conditions are considered:

1. $\mathcal{P}_{2}$ is described by a linear PDE of the form $\mathcal{L} u_{2}=0$, where $\mathcal{L}$ is a linear 
operator.

2. it has no volume non-autonomous forcing terms.

The problem $\mathcal{P}_{2}$ is considered as time independent, when presenting the method hereafter. However, this restriction can be relaxed in several cases. Some examples and numerical experiments will be proposed in section 5 .

The system can be written in strong form as follows:

$$
\begin{array}{r}
\mathcal{F}\left(u_{1}, \partial_{t}^{(\omega)} u_{1}, \nabla_{x}^{(\beta)} u_{1}\right)=0, \text { on } \Omega_{1} \\
h\left(u_{1}\right)=0 \text { on } \partial \Omega_{1} / \Gamma, \\
\mathcal{L} u_{2}=0, \text { on } \Omega_{2} \\
\ell_{2} u_{2}=0, \quad \text { on } \partial \Omega_{2} / \Gamma, \\
f_{1}\left(u_{1}, u_{2}\right)=0, \text { on } \Gamma, \\
f_{2}\left(u_{1}, u_{2}\right)=0, \text { on } \Gamma,
\end{array}
$$

where $\mathcal{F}$ stands for a generic PDE describing the problem $\mathcal{P}_{1}$, depending upon time and space derivatives of maximal orders $\omega$ and $\beta$, respectively. The generic non-homogeneous boundary conditions for $\mathcal{P}_{1}$ are denoted by $h\left(u_{1}\right)=0$. The homogeneous linear boundary conditions for the problem $\mathcal{P}_{2}$ on the boundary $\partial \Omega_{2} / \Gamma$ are expressed by the condition $\ell_{2} u_{2}=0$. The boundary condition $f_{2}$ expressing the coupling conditions for $\mathcal{P}_{2}$ has to be affine in $u_{2}$, while the condition $f_{1}$ for the problem $\mathcal{P}_{1}$ can be generic. The goal is to decouple the problems and to be able to solve $\mathcal{P}_{2}$ efficiently. The main interest, though, is not to have the solution of $\mathcal{P}_{2}$ in the whole domain $\Omega_{2}$. Instead, only an accurate representation of the solution of $\mathcal{P}_{2}$ at the interface $\Gamma$ is needed in order to have a good approximation of the solution of $\mathcal{P}_{1}$. The action of the linear problem $\mathcal{P}_{2}$ on the problem $\mathcal{P}_{1}$ through the coupling conditions can be described by means of the Poincaré-Steklov operator $\mathcal{S}$ defined as follows:

$$
\mathcal{S} v=\mathcal{T} \ell\left(\mathcal{L}^{-1} v\right)
$$

where the quite abstract notation $\mathcal{T} \ell\left(\mathcal{L}^{-1} v\right)$ stands for: solving the linear problem $\mathcal{P}_{2}$ on $\Omega_{2}$ when the datum at the interface is $v$, compute the image of a linear operator of the solution $\ell u_{2}$ and take the trace $\mathcal{T}$ of it.

\subsection{Classical domain decomposition iteration}

The coupled problem described above can be solved by using the Domain Decomposition (DD) method. Since the proposed approach can be seen as a technique to speed up the DD iteration, we recall the basics of this method. A complete review and detailed treatment of the Domain Decomposition methods can be found in $[1,20,21,22]$.

The method relies on the presence of two (or more) subdomains. These can be either physically related to two different physics, as it is the case in the present work, or they can be artificially generated. These subdomains can be chosen 
with or without overlapping. In the present work we consider non-overlapping subdomains. The idea is to couple the dynamics in the two subdomains by enforcing suitable transmission conditions at the interface. Then, the coupling is solved by fixed point iterations. A typical scheme is the Dirichlet to Neumann iteration in its multiplicative version, here applied, for instance, to the Laplace equation:

$$
\begin{cases}-\Delta u_{1}^{k}=0 & \Omega_{1} \\ u_{1}^{k}=u_{2}^{k-1} & \Gamma \\ -\Delta u_{2}^{k}=0 & \Omega_{2} \\ \partial_{n} u_{2}^{k}=\partial_{n} u_{1}^{k} & \Gamma\end{cases}
$$

where $k$ denotes the current domain decomposition iteration and where the boundary conditions on $\partial \Omega$ have been omitted. Other coupling schemes can be considered, such as the Neumann-Neumann and Robin-Robin scheme. Since convergence is not always guaranteed, a relaxation of the Dirichlet condition can be introduced of the form:

$$
u_{1}^{k}=\theta u_{2}^{k-1}+(1-\theta) u_{1}^{k-1} .
$$

With the notation introduced, the problem $\mathcal{P}_{1}$ is defined by $\mathcal{F}=-\Delta u_{1}, \mathcal{P}_{2}$ is also, in this example, a Laplace equation, i.e. $\mathcal{L} u_{2}=-\Delta u_{2}$. The condition for $\mathcal{P}_{1}$ at the interface are given by $f_{1}=u_{1}-u_{2}$ and the condition for the problem $\mathcal{P}_{2}$ are given by $f_{2}=\partial_{n} u_{2}-\partial_{n} u_{1}$. The Poincaré-Steklov operator associated to the problem $\mathcal{P}_{2}$ is defined as:

$$
\begin{array}{r}
\mathcal{S}: H^{-1 / 2}(\Gamma) \rightarrow H^{1 / 2}(\Gamma), \\
\mathcal{S} \partial_{n} u_{1}=u_{2} \quad \text { on } \Gamma
\end{array}
$$

so that $\ell$ is the identity operator on $\Gamma$ and the operator is called the Neumannto-Dirichlet map (N2D).

The discretisation of this example is shown when a finite element basis is used. This provides some insights into the algebraic nature of the discretised Poincaré-Steklov operator, and a motivation for the present work.

Let $b_{i}^{(h)} \in B(\Gamma)$ be a generic finite element function defined on $\Gamma$, then, the matrix $S$ is the representation of the Poincaré-Steklov operator on the basis functions, namely:

$$
S_{i j}:=\left\langle\mathcal{S} b_{j}^{(h)}, b_{i}^{(h)}\right\rangle_{\Gamma},
$$

where $h$ stands for the characteristic mesh length. In such a case, the whole coupled problem admits the following matrix representation:

$$
\left[\begin{array}{ccc}
A_{11} & 0 & A_{1 \Gamma}, \\
0 & A_{22} & A_{2 \Gamma}, \\
A_{\Gamma 1} & A_{\Gamma 2} & A_{\Gamma \Gamma}
\end{array}\right]\left(\begin{array}{l}
u_{1}, \\
u_{2}, \\
u_{\Gamma}
\end{array}\right)=\left(\begin{array}{c}
f_{1}, \\
f_{2}, \\
f_{\Gamma}
\end{array}\right)
$$

After some algebra (see [1] for a detailed derivation), a system for the solution at the interface can be obtained of the form:

$$
\Sigma_{h} u_{\Gamma}=q_{\Gamma},
$$


where $\Sigma_{h}$ is the Schur complement and it is the discrete representation of the Poincaré-Steklov operator. The Schur complement is in general dense and illconditioned for the problems of interest. As a consequence, computing directly the Poincaré-Steklov operator would be very expensive from a computational standpoint, even in the simple case in which both the problems are linear. Moreover, due to the ill-conditioned nature of the matrix, the solution of the system could be affected by large errors. This highlights an important reason to look for a reduced representation of the Poincaré-Steklov operator.

\section{Outline of the method}

The method can be divided, as for the majority of reduced-order modeling methods (see [17] for an overview in the recent advances in this subject), in two phases: an offline phase, and an online one.

\subsection{Offline phase}

In the offline phase, a number of simulations is performed in order to construct a database of meaningful solutions, to be exploited later on and speed up the online phase. Contrary to most of the classical methods of model reduction presented in the literature, we made the choice of simulating only $\mathcal{P}_{2}$ in the offline phase, and not the whole system. This strategy has two main advantages: it allows to perform the simulations in the offline phase without considering the coupling between the two subdomains. The problem $\mathcal{P}_{2}$ is linear and basis functions can be computed in a massive parallel way. Second, from the point of view of the memory usage, there is no need to save the whole solution of $\mathcal{P}_{2}$, but only a restriction of a linear operator applied to its solution on the interface $\Gamma$.

We need to choose a set of basis functions to represent the input datum. Such basis has to be defined on a generic Riemannian manifold, to be orthonormal and complete. In view of these desired properties, a reasonable choice is to a priori take the first $N_{\ell}$ eigenfunctions of the Laplace-Beltrami operator defined on the surface, with $N_{\ell} \ll N_{\Gamma}$, the number of degrees of freedom at the interface. The advantages of choosing the eigenfunctions of the Laplace-Beltrami operator are the following:

1. The basis is a complete basis of $V:=L_{2}(\Gamma)$.

2. It is hierarchical.

3. It automatically accounts for symmetries in the geometry.

4. The extraction of the basis amounts to solve a sparse eigenvalue problem defined on $\Gamma$.

5. On particular (but meaningful) geometrical settings, the basis coincides with the eigenfunctions of the Poincaré-Steklov operator (see Appendix A). 
Let the eigenfunctions of the Laplace-Beltrami operator be denoted as follows: $v_{i} \in V, i=1, \ldots, N_{\ell}$ is such that $-\Delta_{\Gamma} v_{i}=\mu_{i} v_{i}$.

The problem in weak form reads:

$$
\left\langle\nabla_{\Gamma} v_{i}, \nabla_{\Gamma} \omega\right\rangle_{\Gamma}=\mu_{i}\left\langle v_{i}, \omega\right\rangle_{\Gamma}, \forall \omega \in H^{1}(\Gamma)
$$

where $\nabla_{\Gamma}$ denotes the surface gradient and $\langle u, v\rangle_{\Gamma}=\int_{\Gamma} u v d \Gamma$ is the inner product on the interface $\Gamma$. The problem is then discretised by using $P_{1}$ finite elements.

Once the basis has been extracted by solving a sparse eigenvalue problem, the problem $\mathcal{P}_{2}$ is solved, for every input function $v_{i}$. Only the image of a linear operator applied to the solution is stored on the boundary, i.e.: $y_{i}=\mathcal{S} v_{i}=$ $\mathcal{T} \ell\left(u_{2}^{(i)}\right)$, where $u_{2}^{(i)}=\mathcal{L}^{-1}\left(v_{i}\right)$.

In the case in which the boundary conditions on $\partial \Omega_{2} / \Gamma$ are not homogeneous, other problems can be solved in the offline phase to account for the contribution of the terms on $\partial \Omega_{2} / \Gamma$ at the interface.

\subsection{Online phase}

The action of the Poincaré-Steklov operator on a generic input datum $d$ can be approximated as follows:

$$
\mathcal{S} d \approx \mathcal{S}_{0}+\sum_{j}^{N_{\ell}}\left\langle d, v_{j}\right\rangle_{\Gamma} y_{j}+\sum_{k}^{N_{o}^{(n)}}\left\langle d, w_{k}\right\rangle_{\Gamma} z_{k},
$$

where $\mathcal{S}_{0}$ accounts for eventual non-homogeneous boundary conditions for $\mathcal{P}_{2}$ on $\partial \Omega_{2} / \Gamma$, the second term corresponds to the contribution of the projection of the datum $d$ in the space spanned by the Laplace-Beltrami eigenfunctions and the last term is an online update of the basis, such that $\left\langle w_{k}, y_{j}\right\rangle_{\Gamma}=0$. The online update of the basis is similar, in the spirit, to what is proposed in $[18,19]$. In particular, when an error criterion is not fulfilled, the basis is updated by adding elements to it, coming, in the present approach, from a full-order problem simulation. The main difference with respect to the cited works concerns the way the update is performed. In the present approach we decided not to use a thin SVD update, but simply to increase the basis size and perform an orthogonalisation through a Modified Gram Schmidt (MGS). This proved to be sufficient for the purposes of this work. The online update is performed as follows. Consider a generic time instant $t^{(n)}$. The approximation space in use is $V \cup W^{(n)}$, that is, the union of the set of the Laplace-Beltrami eigenfunctions and the set of the $N_{o}^{(n)}$ online basis functions possibly added in the previous time iterations. Once the datum $d^{(n)}$ is available, coming from $\mathcal{P}_{1}$, its reconstruction error on the space in use is:

$$
\varepsilon_{d}^{(n)}:=\left\|d^{(n)}-\sum_{i=1}^{N_{\ell}}\left\langle d^{(n)}, v_{i}\right\rangle_{\Gamma} v_{i}-\sum_{j=1}^{N_{o}^{(n)}}\left\langle d^{(n)}, w_{j}\right\rangle_{\Gamma} w_{j}\right\|_{\Gamma, 2}
$$


Let $\bar{\varepsilon}_{d}$ be the tolerance chosen for the datum representation. If $\varepsilon_{d}^{(n)}>\bar{\varepsilon}_{d}$ then an online update is needed. Let the basis function to be added be:

$$
w_{N_{o}^{(n)}+1=N_{o}^{(n+1)}}=C\left(d^{(n)}-\sum_{i=1}^{N_{\ell}}\left\langle d^{(n)}, v_{i}\right\rangle_{\Gamma} v_{i}-\sum_{j=1}^{N_{o}^{(n)}}\left\langle d^{(n)}, w_{j}\right\rangle_{\Gamma} w_{j}\right),
$$

where $C$ is the normalisation constant, chosen such that: $\left\|w_{N_{o}^{(n+1)}}\right\|_{\Gamma, 2}=1$. This step is performed through an MGS method. The corresponding output $z_{N_{o}^{(n+1)}}$ is computed by actually solving the problem $\mathcal{P}_{2}$. The main advantage of the online enrichment is that a higher precision is guaranteed. Theoretically, the online update could be required at all the evaluations of the secondary problem, but in practice, see also the numerical section, only a few online solution of $\mathcal{P}_{2}$ are required. In fact, the real drawback of using the online update is that the access to the high fidelity model for $\mathcal{P}_{2}$ is required both in the online and in the offline phase. For this reason, depending on the way in which the software is implemented and on the size of $\mathcal{P}_{2}$, one may decide to skip the online phase and to rely only on the offline set of basis functions.

\subsection{Retrieving the Poincaré-Steklov eigenfunctions}

Once the output of $\mathcal{P}_{2}$ have been computed for all the basis functions on $\Gamma$, the eigenfunctions of the Poincaré-Steklov operator, if needed, can be retrieved. This allows to perform a Low Rank Decomposition of the operator that can be exploited in parametric Domain Decomposition methods. In particular, let the eigenfunctions of the Poincaré-Steklov operator be written as a linear combination of the Laplace-Beltrami eigenfunctions as:

$$
\hat{\varphi}_{i}=\sum_{j=1}^{N_{\ell}} U_{i j} v_{j}
$$

where $\hat{\varphi}_{i}$ is the approximation of the Poincaré-Steklov operator eigenfunction, $U$ is the representation of the Poincaré-Steklov eigenfunctions onto the LaplaceBeltrami eigenfunctions. The equation $\mathcal{S} \varphi_{i}=\lambda_{i} \varphi_{i}$, when discretized on the first $N_{\ell}$ eigenfunctions $v_{j}$, becomes:

$$
\sum_{j=1}^{N_{\ell}} U_{i j} \mathcal{S} v_{j}=\hat{\lambda}_{i} \sum_{j=1}^{N_{\ell}} U_{i j} v_{j}
$$

This is projected onto the space spanned by the eigenfunctions of the LaplaceBeltrami operator leading to:

$$
\sum_{j=1}^{N_{\ell}} U_{i j}\left\langle\mathcal{S} v_{j}, v_{k}\right\rangle_{\Gamma}=\hat{\lambda}_{i} \sum_{j=1}^{N_{\ell}} U_{i j} \delta_{j k}
$$


This is a reduced eigenvalue problem for the representation of the PoincaréSteklov eigenfunctions on the Laplace-Beltrami ones (namely $U_{i j}$ ). The problem can be recasted as follows:

$$
S U=U \Lambda,
$$

where $S_{j k}:=\left\langle\mathcal{S} v_{j}, v_{k}\right\rangle_{\Gamma}=\left\langle y_{j}, v_{k}\right\rangle_{\Gamma}$ is the representation of the Poincaré-Steklov operator onto the Laplace-Beltrami eigenfunction basis.

Having an eigenbasis for the operator $\mathcal{S}$ (think for instance to the N2D map) allows to easily recover the action of other maps on the data. Indeed, the eigenfunctions of all the maps coincide and only the spectrum changes and it can be recovered via a purely algebraic relation. This can be useful when dealing with DD iterations in which a Robin condition has a possibly varying parameter (see [23]) or if the scenario to be simulated changes on a given geometrical setting. In all these cases, it is not necessary to re-compute the offline phase, so that no extra computational cost is involved.

Remark: The proposed method can be applied straightforwardly to systems whose geometry is fixed (corresponding to a single scenario for $\mathcal{P}_{2}$ ). An interesting perspective we briefly prospect on in this remark concerns the cases in which multiple scenarios are involved. This is for instance the case in which the interface depends upon a set of parameters $\boldsymbol{\mu}$. This leads to a parametric representation of the Poincaré-Steklov operator. To compute a low-rank decomposition of the operator, the M-DEIM method introduced in [24] could be used. The authors introduced it to deal with problems that, after discretization, are featured by matrices that depend on parameters in a non-affine way. In the present context, given a sample of $\boldsymbol{\mu}$, the corresponding Laplace-Beltrami eigenfunction can be computed and the outputs of $\mathcal{P}_{2}(\boldsymbol{\mu})$ stored. These can be used to approximate, in the online phase, the action of $\mathcal{S}(\boldsymbol{\mu})$ on the input.

\section{Numerical Analysis}

In this section an analysis of the approximation properties of the method and its computational costs is detailed.

\subsection{Properties of Poincaré-Steklov operator}

Here we recall some properties of the Poincaré-Steklov operator in the case in which the problem $\mathcal{P}_{2}$ is linear elliptic and symmetric (see [25]). The numerical analysis of the method will be performed by assuming the same hypotheses. The numerical experiments, instead, will be presented in a more general setting.

In what follows, we denote the Neumann to Dirichlet map (N2D) by $S$. In this case, $f_{2}=\partial_{n} u_{2}-d\left(u_{1}\right)$, where $d\left(u_{1}\right)$ is the datum coming from $\mathcal{P}_{1}$. The operator $S$ is defined from $H^{-1 / 2}(\Gamma)$ to $H^{1 / 2}(\Gamma)$ and it is the trace of the solution of $\mathcal{P}_{2}$. The corresponding inverse map $S^{-1}$ is the Dirichlet to Neumann map (D2N), it takes elements from $H^{1 / 2}(\Gamma)$ and its result is in $H^{-1 / 2}(\Gamma)$. In 
this case $f_{2}=u_{2}-d\left(u_{1}\right)$ and the application of the map provides the normal derivative of $u_{2}$ on $\Gamma$.

The operator $S$ is compact in $L^{2}(\Gamma)$, continuous and symmetric with respect to the $L^{2}(\Gamma)$ duality between $H^{-1 / 2}(\Gamma)$ to $H^{1 / 2}(\Gamma)$ (see [25] for the proof of these properties). Both $S$ and $S^{-1}$ are positive definite and they both induce a norm on $H^{-1 / 2}(\Gamma)$ and $H^{1 / 2}(\Gamma)$, respectively.

In this case, the eigenfunctions of $S$ are a complete orthonormal basis of $L_{2}(\Gamma)$.

\subsection{Convergence}

In this section, the convergence of the proposed method is investigated in the case in which $\mathcal{P}_{2}$ is defined by a linear elliptic and symmetric operator, enjoying the properties introduced in section 4.1. In what follows, for sake of compactness in the notation, the standard $H^{s}(\Gamma)$ norm will be denoted by $\|\cdot\|_{s}$, where it is intended that $\|\cdot\|_{0}$ is the standard $L_{2}(\Gamma)$ norm. The result is summarised in the following proposition.

Proposition 1. Let $\partial \Omega_{2}$ be $C^{2}$ and let $S: H^{-1 / 2}(\Gamma) \rightarrow H^{1 / 2}(\Gamma)$ be the N2D map, $S^{-1}$ the D2N map; let $w$ be the datum and $\hat{w}$ its projection onto the subset of the first $N_{\ell}$ Laplace-Beltrami eigenfunctions. Then:

1. if $w \in L_{2}(\Gamma)$, then $\lim _{N_{\ell} \rightarrow \infty}\|S(w-\hat{w})\|_{0}=0$.

2. if $w \in H^{2}(\Gamma)$, then: $\|S(w-\hat{w})\|_{0} \leq C_{1} N_{\ell}^{-2 / d_{\Gamma}}|w|_{2}$.

3. if $w \in H^{2}(\Gamma)$, then: $\left\|S^{-1}(w-\hat{w})\right\|_{0} \leq C_{2} N_{\ell}^{-1 / 2 d_{\Gamma}}|w|_{2}$.

Proof. In order to prove the first point, the following inequality is considered:

$$
\|S(w-\hat{w})\|_{0} \leq \lambda_{\max }\|w-\hat{w}\|_{0},
$$

where $\lambda_{\max }$ is the largest eigenvalue of the N2D map. Since the LaplaceBeltrami eigenfunctions are a complete basis of $L_{2}(\Gamma)$, the convergence follows. The second point can be proved by considering the approximation properties of the Laplace-Beltrami eigenfunctions (see for instance [26, 27]). Under the hypothesis that $w$ satisfies the same boundary conditions as the eigenfunctions basis on $\partial \Gamma$, it holds:

$$
\|w-\hat{w}\|_{0} \leq c N_{\ell}^{-2 / d_{\Gamma}}|w|_{2},
$$

Thus: $C_{1}=\lambda_{\max } c$, where $c$ is a constant depending on the domain $\Gamma$.

The proof of the convergence for the inverse map is more delicate since the map causes a loss of regularity. Let us assume that the datum $w \in H^{2}(\Gamma)$. In this case, see [25]:

$$
S^{-1}(w-\hat{w}) \in H^{1 / 2}(\Gamma) .
$$


Then, thanks to the trace inequality and the regularity of the Laplace problem (see [28] for a detailed derivation), the following chain of inequalities holds:

$\left\|S^{-1}(w-\hat{w})\right\|_{0} \leq B_{1}\left\|S^{-1}(w-\hat{w})\right\|_{1 / 2} \leq C_{T} B_{1}\|u\|_{H^{2}\left(\Omega_{2}\right)} \leq C_{T} B_{1} B_{2}\|(w-\hat{w})\|_{3 / 2}$,

where $u$ is the Dirichlet harmonic extension of $w-\hat{w}, C_{T}, B_{1}, B_{2}$ are constants that depend on $\Gamma\left(B_{1}\right)$ and on $\Omega_{2}\left(C_{T}, B_{2}\right)$. By the properties of interpolation of Sobolev spaces, see [29], the last term satisfies:

$$
\|(w-\hat{w})\|_{3 / 2} \leq B_{3}\|(w-\hat{w})\|_{2}^{3 / 4}\|(w-\hat{w})\|_{0}^{1 / 4},
$$

where $B_{3}$ is a constant depending on $\Gamma$. This is sufficient at once to prove the convergence and to derive, thanks to the approximation properties of the Laplace eigenfunctions, the theoretical rate, as done for the map $S$.

The result of the proposition shows that the method converges. When the N2D map is approximated, in a 3D problem, a linear (in the number of eigenfunctions) convergence is expected. When the inverse map (D2N) is approximated, due to the loss of regularity induced by the map, a potentially slower convergence rate is obtained. In the very specific case of a solution $w$ such that its higher order derivatives satisfy the same boundary conditions as the eigenfunctions basis on $\partial \Gamma$ we have exponential convergence (see for instance $[26,27])$.

\subsubsection{A semi-analytical example.}

In this subsection a computation is shown in order to compute the rate of convergence of the method in a simple setting. Consider for instance the problem $\mathcal{P}_{2}$ defined as $-\Delta u=0$ on a unit square $[0,1]^{2}$ with Dirichlet boundary conditions on three sides and a datum imposed on $y=0$. Let the datum $d$ be expanded in a Fourier series and let $\hat{d}_{k}$ be the $k$-th coefficient. Then, the N2D and the D2N maps can be computed analytically:

$$
\begin{aligned}
S d & =\sum_{k}^{\infty} \sqrt{2} \hat{d}_{k} \frac{\tanh (\pi k)}{\pi k} \sin (\pi k x), \\
S^{-1} d & =\sum_{k}^{\infty} \sqrt{2} \hat{d}_{k} \frac{\pi k}{\tanh (\pi k)} \sin (\pi k x) .
\end{aligned}
$$

In the last two equations the regularising effect of $S$ is evident: the Fourier coefficients of the image are going to zero faster with respect to those of the datum. On the other hand, for $S^{-1}$, the coefficients are multiplied by $k$ and therefore they tend to zero more slowly. When computing the truncation error committed by retaining only $k=N_{\ell}$ space frequencies, the following expression 
is obtained:

$$
\begin{gathered}
\varepsilon_{S}=\|S(d-\hat{d})\|_{2}=\left(\sum_{k>N_{\ell}} \hat{d}_{k}^{2} \tanh (k \pi)^{2} \pi^{-2} k^{-2}\right)^{1 / 2}, \\
\varepsilon_{S^{-1}}=\left\|S^{-1}(d-\hat{d})\right\|_{2}=\left(\sum_{k>N_{\ell}} \hat{d}_{k}^{2} \pi^{2} k^{2} \tanh (k \pi)^{-2}\right)^{1 / 2},
\end{gathered}
$$

where $\hat{d}$ is the projection of the datum on the first $N_{\ell}$ basis functions.

In Table 1 we reported the estimated rate of decay of the Fourier coefficients $\hat{d}_{k}$ and the $L_{2}$-norm of the errors for the two maps, for two different functions,

\begin{tabular}{|c|c|c|c|c|c|}
\hline$d$ & & Regularity & $\hat{d}_{k}$ & $\left\|\varepsilon_{S}\right\|_{0}$ & $\left\|\varepsilon_{S^{-1}}\right\|_{0}$ \\
\hline$\left\{\begin{array}{l}0 \\
\frac{x^{2}}{2}-\frac{x}{5}+\frac{1}{50} \\
-\frac{x^{2}}{2}+\frac{3 x}{5}-\frac{7}{50} \\
\frac{x^{2}}{2}-x+\frac{1}{2}\end{array}\right.$ & $\begin{array}{l}0 \leq x<1 / 5 \\
1 / 5 \leq x<2 / 5 \\
2 / 5 \leq x<4 / 5 \\
4 / 5 \leq x \leq 1\end{array}$ & $H^{2}(0,1)$ & $k^{-3}$ & $k^{-3.5}$ & $k^{-1.5}$ \\
\hline$\left\{\begin{array}{l}3 x+1 \\
1 \\
2-2 x\end{array}\right.$ & $\begin{array}{l}0 \leq x<1 / 3 \\
1 / 3 \leq x<1 / 2 \\
1 / 2 \leq x \leq 1\end{array}$ & $H^{1}(0,1)$ & $k^{-2}$ & $k^{-2.5}$ & $k^{-0.5}$ \\
\hline
\end{tabular}
characterized by a different regularity. The results show that the convergence,

Tab. 1: Semi-analytical estimation of the rate of convergence of the method.

in this case, is faster than expected. In particular, the result of the proof is pessimistic since in this case the Poincaré-Steklov operator eigenfunctions coincide with the Laplace-Beltrami eigenfunctions at the interface.

\subsection{Theoretical analysis of the computational costs.}

The computational cost of the procedure and the theoretical speed-up are presented. Let $\mathcal{N}_{1}, \mathcal{N}_{2}$ be the number of degrees of freedom of $\mathcal{P}_{1}$ and $\mathcal{P}_{2}$ respectively when they are discretised by means of a standard Finite Element method. Then, let $I_{d d}$ be the average number of iterations of Domain Decomposition. We assume that the computational costs associated to the solution of an average iteration of Domain Decomposition amounts to:

$$
\mathcal{C}_{I} \approx I_{d d}\left(C_{1} \mathcal{N}_{1}^{\alpha_{1}}+C_{2} \mathcal{N}_{2}^{\alpha_{2}}\right),
$$

where the $\alpha_{i}$ depend on the solvers chosen for the the two problems. When the GMRES method is used (without preconditioning) a quadratic behavior is expected $\left(\alpha_{i} \sim 2\right)$. Other state of the art solvers, e.g. multigrid, shows approximately linear convergence $\left(\alpha_{i} \sim 1\right.$, see for instance [30]). Let $\mathcal{N}_{\Gamma}$ be the 
number of degrees of freedom at the interface. Then, the method consists of approximating the result of $\mathcal{P}_{2}$ by projecting the datum at the interface onto the basis and to reconstruct the output of the Poincaré-Steklov operator, at the interface. Let $N_{\ell}$ be the number of modes. This operation has a computational cost, denoted by $\mathcal{C}_{r}$ of:

$$
\mathcal{C}_{r} \approx 3 \mathcal{N}_{\Gamma} N_{\ell}+\mathcal{O}(1)
$$

When applying the method, the average Domain Decomposition iteration has a reduced cost $\left(\mathcal{C}_{R}\right)$ of:

$$
\mathcal{C}_{R} \approx I_{r d d}\left(C_{1} \mathcal{N}_{1}^{\alpha_{1}}+3 \mathcal{N}_{\Gamma} N_{\ell}\right)+\mathcal{O}(1)
$$

where the average number of Domain Decomposition iteration $I_{r d d}$ may vary by virtue of a regularizing effect of the reduced-order model.

Few comments are in order. The speed up in the solution of the problem $\mathcal{P}_{2}$ is expected to be very large. Indeed, it is the ratio between a term that scales as the $\alpha_{2}$-power of the number of degrees of freedom in the domain $\Omega_{2}$ and a term which is linear in the number of degrees of freedom of the interface. The overall speed-up of a single iteration is due to two contributions: the acceleration of the solution of $\mathcal{P}_{2}$ and a possible regularizing effect:

$$
\frac{\mathcal{C}_{I}}{\mathcal{C}_{R}} \approx \frac{I_{d d}\left(C_{1} \mathcal{N}_{1}^{\alpha_{1}}+C_{2} \mathcal{N}_{2}^{\alpha_{2}}\right)}{I_{r d d}\left(C_{1} \mathcal{N}_{1}^{\alpha_{1}}+3 \mathcal{N}_{\Gamma} N_{\ell}\right)},
$$

so that the method is very efficient when the number of degrees of freedom of $\mathcal{P}_{2}$ is large. By observing Eq.(35) it is clear that the best conditions for expecting a significant speed up are: $\mathcal{N}_{2} \gg \mathcal{N}_{\Gamma}$ and $\mathcal{N}_{2} \gg \mathcal{N}_{1}$. In fact, the first condition guarantees a speed up in the solution of $\mathcal{P}_{2}$ and the second condition guarantees that $\mathcal{P}_{2}$ was the most costly part of the simulation. In other cases where the second condition is not satisfied, but we have, for instance, $\mathcal{N}_{2}=\mathcal{N}_{1}$ the total solution cost will be reduced only by a factor two. Remark that in the case in which there are several systems in interaction, the speed up of the method is expected to be larger and larger.

The total cost of performing $N_{\text {sim }}$ simulations each of which has $N_{T}$ time steps can be roughly expressed, for the Full-order method $\left(\mathcal{C}_{D D}\right)$ and for the Reduced-Order one $\left(\mathcal{C}_{R O M}\right)$ as:

$$
\begin{array}{r}
\mathcal{C}_{D D} \approx N_{s i m} N_{T} \mathcal{C}_{I} \\
\mathcal{C}_{R O M} \approx N_{s i m} N_{T} \mathcal{C}_{R}+N_{\ell} C_{2} \mathcal{N}_{2}^{\alpha_{2}}+6 N_{\ell}^{2} \mathcal{N}_{\Gamma}
\end{array}
$$

where the last term accounts for the overheads of the offline phase, consisting in solving $N_{\ell}$ problems $\mathcal{P}_{2}$ and by computing $N_{\ell}$ eigenfunctions of the LaplaceBeltrami operator defined at the interface.

When the online update of the basis is used to reduce the error in the computation, an extra cost has to be accounted for, consisting of solving one $\mathcal{P}_{2}$ problem, whose cost is $\propto \mathcal{C}_{2} \mathcal{N}_{2}^{\alpha_{2}}$ and a Modified Gram-Schmidt orthonormalisation for the input function at the interface. 


\section{Numerical Experiments}

In this section three numerical experiments are shown, on systems characterised by different interactions and geometrical settings. The first two testcases were mainly motivated by the study of physiological flows, think for instance of blood vessels surrounded by soft tissues, and are models of fluid-fluid interaction. The last test is, instead, a paradigmatic example of fluid-structure interaction applications arising in an industrial context. Although the method is tested on fluid-fluid or fluid-structure interaction problems, we would like to emphasize that it finds application in a broad range of engineering problems.

In particular, the first test case is a simple example where the external problem is described by a Laplacian operator and where Proposition 2 holds. The second test case is used to quantitavely confirm the convergence properties derived in the previous section and to test the online enrichment strategy. The third and last test case involves a more complex operator for the external problem, which is a stationary Stokes problem, and it is used to measure the speed up.

\subsection{Incompressible flow interacting with a porous medium}

In this testcase, the first system $\mathcal{P}_{1}$ is governed by the Navier-Stokes equations in interaction with a porous medium, $\mathcal{P}_{2}$, described by the Darcy equation. A full analysis of this coupling and of suitable domain-decomposition schemes can be found in [31]. The geometrical setting is the following: the domain $\Omega=$ $\Omega_{1} \cup \Omega_{2}$ is a parallelepiped $(0,10) \times(0,5) \times(0,10)$ divided in two parallelepipeds $\Omega_{1}=(0,10) \times(0,5) \times(9,10)$ and $\Omega_{2}=(0,10) \times(0,5) \times(0,9)$ (see Fig. 1$)$. The two flows are separated by the planar interface $\Gamma=(0,10) \times(0,5) \times\{9\}$. In $\Omega_{1}$, the fluid is described by the time-dependent Navier-Stokes equations: $\partial_{t} \boldsymbol{u}+\boldsymbol{u} \cdot \nabla \boldsymbol{u}=\nabla \cdot \boldsymbol{\sigma}\left(\boldsymbol{u}, p_{1}\right), \nabla \cdot \boldsymbol{u}=0$, where $\boldsymbol{\sigma}\left(\boldsymbol{u}, p_{1}\right)=\nu\left(\nabla \boldsymbol{u}+\nabla \boldsymbol{u}^{T}\right)-p_{1} \boldsymbol{I}$ is the Cauchy stress tensor, $\nu=0.04$ the fluid viscosity and $\boldsymbol{u}$ and $p_{1}$ the fluid velocity and pressure. We denote by $\boldsymbol{n}_{1}$ the outward normal with respect to $\Omega_{1}$ and by $\boldsymbol{n}_{2}$ the normal oriented outward with respect to $\Omega_{2}$.

For the boundary conditions at $\partial \Omega_{1} \backslash \Gamma$, we assigned a non-homogeneous natural condition, $\boldsymbol{\sigma}(\boldsymbol{u}, p) \boldsymbol{n}_{1}=-10 \sin (0.5(x+y)-100 t)$, on $\Gamma_{i n}=(0,10) \times$ $(0,5) \times\{z=10\}$, the top surface of $\Omega_{1}$. On the lateral surface, $\Gamma_{\text {lat }, 1}=\partial \Omega_{1} \backslash$ $\left(\Gamma \cup \Gamma_{i n}\right)$, mixed homogeneous boundary conditions were imposed on the normal velocity and on the tangential component of the normal stress. Moreover, the tangential velocity was set to zero at the interface $\Gamma$.

In the second compartment, $\Omega_{2}$, the flow in the porous medium is described by the Darcy's equation: $-\operatorname{div}\left(K \nabla p_{2}\right)=0$, where $K=0.2$ is the permeability which was assumed to be a scalar.

On $\partial \Omega_{2} \backslash \Gamma$ we imposed the following boundary conditions: zero pressure at $\Gamma_{\text {out }}=(0,10) \times(0,5) \times\{z=0\}$ and homogenous Neumann conditions on $\Gamma_{\text {lat }, 2}=\partial \Omega_{2} \backslash\left(\Gamma \cup \Gamma_{\text {lat }, 2}\right)$.

The two systems are coupled by the following interface conditions on the 

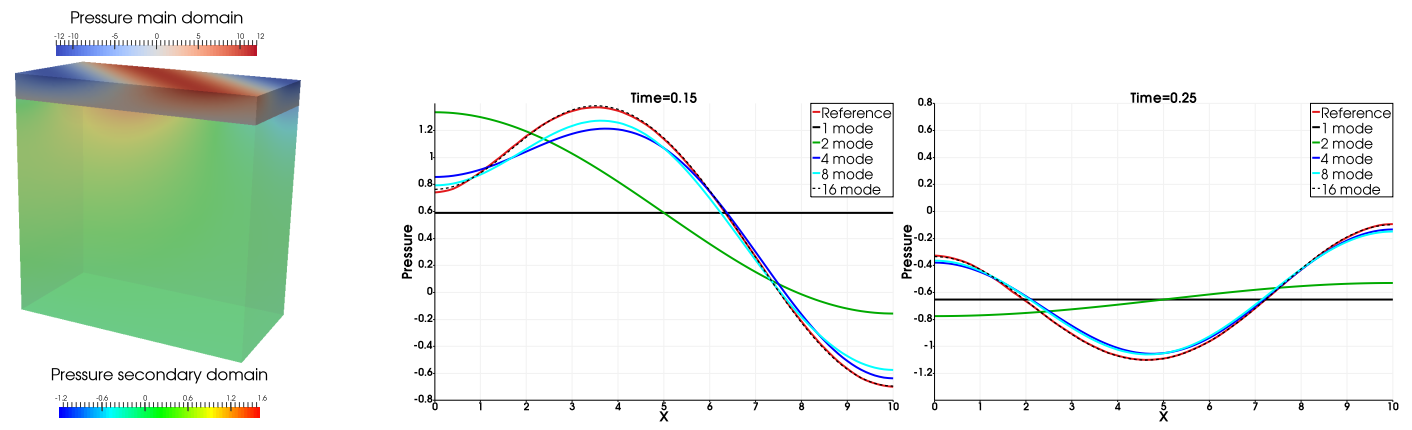

Fig. 1: A snapshot of the reference solution for $t=0.15$ (left panel). Pressure $p$ at the interface $\Gamma$ (first test case) along the line $y=2.5$ for two different time instants (center and right panel). Finite elements results are compared with the reduced approach for different numbers of modal functions.

normal direction:

$$
\begin{cases}\boldsymbol{\sigma}(\boldsymbol{u}, p) \boldsymbol{n}_{1} \cdot \boldsymbol{n}_{1}=-p_{2} & \Gamma \\ \nabla p_{2} \cdot \boldsymbol{n}_{2}=\frac{1}{K} \boldsymbol{u} \cdot \boldsymbol{n}_{1} & \Gamma .\end{cases}
$$

To solve the problem with an iterative scheme the following domain decomposition scheme is adopted:

$$
\begin{cases}\boldsymbol{\sigma}\left(\boldsymbol{u}^{k}, p^{k}\right) \boldsymbol{n}_{1} \cdot \boldsymbol{n}_{1}+\alpha \boldsymbol{u}^{k}=-p_{2}^{k-1}+\alpha \boldsymbol{u}^{k-1} & \Gamma \\ \nabla p_{2}^{k} \cdot \boldsymbol{n}_{2}=\frac{1}{K} \boldsymbol{u}^{k} \cdot \boldsymbol{n}_{1} & \Gamma,\end{cases}
$$

where $k$ denotes the current domain decomposition iteration and $\alpha=0.1$ is a relaxation parameter. A standard Aitken acceleration is used to reduce the number of fixed point iterations (see [3]). The Steklov operator is therefore a Neumann to Dirichlet map in this case. We also remark that, in such a simple geometry, the eigenfunctions of the Steklov and those of the Laplace-Beltrami operators coincide, see Appendix A.

To discretise the system in space, P1-P1 stabilized finite elements are used. The computational mesh consists of 59268 tetrahedra for $\Omega_{1}$ and 439458 for $\Omega_{2}$, while on $\Gamma$ there are 2776 degrees of freedom and the time step $\Delta t$ is equal to 0.01 .

The result of the reduction are shown in Figure 1, where the pressure $p$ is displayed at the interface and the reduced solutions are compared to the reference one for two different time instants. We observe that, in this case, with one and two modes the behavior of the system substantially differs from the reference one. However, by adding few modes the convergence to the reference dynamics is quite fast. 


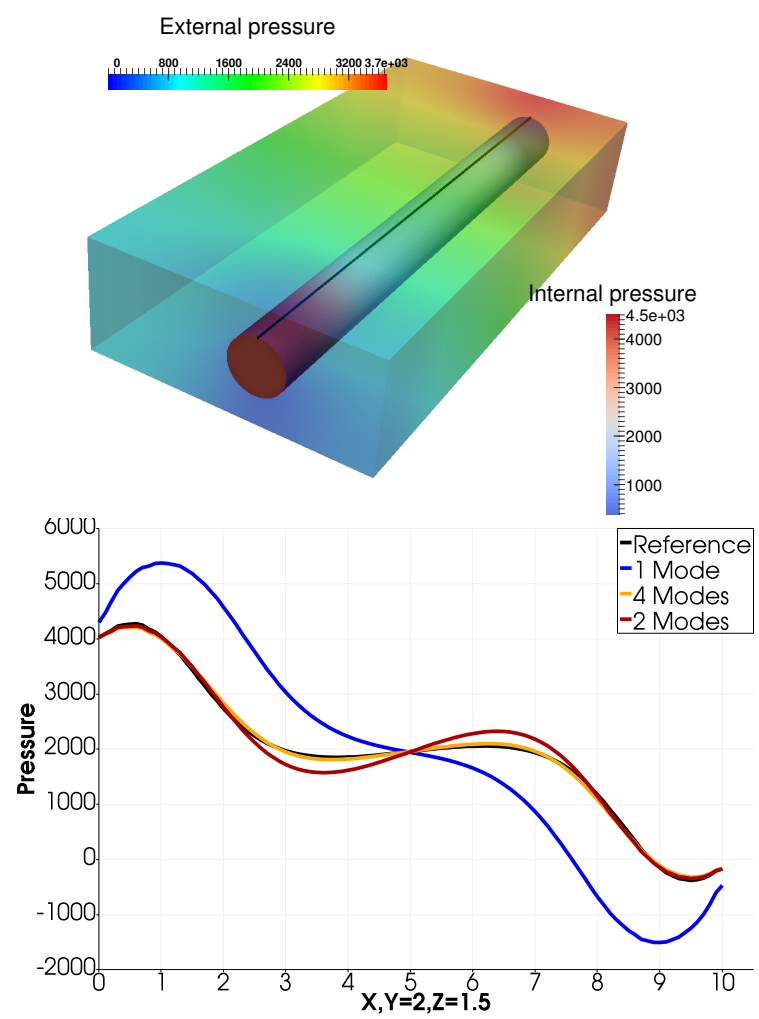

Fig. 2: On the left panel, we have a snapshot of the reference solution for $t=$ 0.006 . The tube is the domain $\Omega_{1}$ where we solve the Stokes equations. On the right panel pressure $p_{1}$ at the black line of the left panel has been displayed for different choices of the offline basis' dimension that are compared to the reference solution.

\subsection{A tube embedded in an inviscid fluid flow}

The second testcase models a more complex system: $\mathcal{P}_{1}$ is a non-steady Stokes flow in a thin elastic tube, coupled with an external inviscid non-steady flow $\left(\mathcal{P}_{2}\right)$. The geometry of the test is shown in Figure 2. The goal is twofold: to provide an example on how the hypothesis of time independence of $\mathcal{P}_{2}$ can be relaxed and to test the method robustness when dealing with ill-posed decoupled problems.

The whole domain is, as in the first test, a parallelepiped $\Omega=(0,10) \times$ $(0,5) \times(0,2)$. The first subdomain $\Omega_{1}$ is the cylinder $(0,10) \times \Gamma_{i n}$ with $\Gamma_{i n}$ being the circle in the $y z$ plane centered in $(x=0, y=2, z=1)$ and with radius $r=0.5, \Gamma_{\text {out }}$ denotes the corresponding face in $x=10$. The interface between the two domains is denoted by $\Gamma$ and it is the lateral surface of $\Omega_{1}$. The domain $\Omega_{2}=\Omega \backslash \Omega_{1}$ is the remaining portion of $\Omega$. 
The problem $\mathcal{P}_{1}$ is the Stokes system and, at the interface $\Gamma$, we added an elastic shell to model the tube elasticity. For the sake of simplicity, we present the system in its discretised form, at time $t_{n}=n \Delta t$ :

$$
\mathcal{P}_{1} \begin{cases}\frac{1}{\Delta t} \boldsymbol{u}^{n}-\nu \Delta \boldsymbol{u}^{n}+\nabla p^{n}=\frac{1}{\Delta t} \boldsymbol{u}^{n-1} & \Omega_{1} \\ \nabla \cdot \boldsymbol{u}^{n}=0 & \Omega_{1} \\ \boldsymbol{\sigma}\left(\boldsymbol{u}^{n}, p_{1}^{n}\right) \boldsymbol{n}_{1}=-p_{\text {in },(\text { out })}\left(t_{n}\right) & \Gamma_{\text {in, (out })} \\ \boldsymbol{\sigma}\left(\boldsymbol{u}^{n}, p_{1}^{n}\right) \boldsymbol{n}_{1}+\mathcal{L}\left(\boldsymbol{u}^{n}\right)=\mathcal{F}^{n-1, n-2}-p_{2}^{n} \boldsymbol{n}_{1} & \Gamma \\ \eta^{n}=\eta^{n-1}+\Delta t \boldsymbol{u}^{n} \cdot \boldsymbol{n}_{1} & \Gamma,\end{cases}
$$

where $p_{\text {in }}(t)$ is a given function of time and $p_{\text {out }}=0$. The membrane is rendered by two terms $\mathcal{L}\left(\boldsymbol{u}^{n}\right)$ and $\mathcal{F}$ in the boundary condition, the first one being a symmetric operator on $\Gamma$ and the second being a function of the values of the solution at the previous time steps. The structure equation is embedded in this two terms and the displacement is then computed through an algebraic equation. The resulting boundary condition is a generalized Robin condition. It is beyond the scope of this work to get into the details of this simplified fluid-structure interaction model; a detailed derivation can be found in [32].

The equation for $\mathcal{P}_{2}$ describes a time-dependent inviscid fluid flow of the form:

$$
\begin{cases}\rho_{2} \partial_{t} \boldsymbol{u}_{2}=-\nabla p_{2} & \Omega_{2} \\ \nabla \cdot \boldsymbol{u}_{2}=0 & \Omega_{2} \\ \boldsymbol{u}_{2}=0 & \partial \Omega_{2} \backslash \Gamma \\ \boldsymbol{u}_{2}=\boldsymbol{u} & \Gamma .\end{cases}
$$

Remark that this is an example in which the working hypothesis on $\mathcal{P}_{2}$ are not fulfilled at continuous level. However, after time discretisation, this problem can be solved by the proposed approach. Indeed, we use an implicit Euler scheme by substituting $\partial_{t} \boldsymbol{u}_{2} \sim \frac{\boldsymbol{u}_{2}^{n}-\boldsymbol{u}_{2}^{n-1}}{\Delta t}$, then we apply the divergence operator to the resulting momentum equation obtaining:

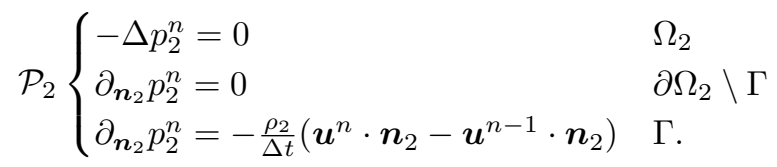

The problem $\mathcal{P}_{2}$, decoupled from $\mathcal{P}_{1}$, is an ill-posed problem. Hence, a relaxed interface condition is used:

$$
\partial_{\boldsymbol{n}_{2}} p_{2}^{n, k}+\alpha p_{2}^{n, k}=-\frac{\rho_{2}}{\Delta t}\left(\boldsymbol{u}^{n, k} \cdot \boldsymbol{n}_{2}-\boldsymbol{u}^{n-1, \infty} \cdot \boldsymbol{n}_{2}\right)+\alpha p_{2}^{n, k-1},
$$

where the $k$ refers to the current domain decomposition iteration.

The solution of this system is featured by counter-propagating elastic waves at the interface $\Gamma$. When the wave has a short wavelength it is expected that its approximation requires a large number of Laplace-Beltrami eigenfunctions. Therefore, this test is a good benchmark to investigate the performance of the 
method both in its purely offline version and with the online update correction. The results of this comparison are reported in Figure 3. In the left and center panel, the error with respect to the reference solution at the interface and in the whole domain are shown in norm $L^{2}\left((0, T) ; L^{2}(\Gamma)\right)$ and $L^{2}\left((0, T) ; L^{2}\left(\Omega_{1}\right)\right)$ , as a function of the number of modes, both without (purely offline) and with the online update of the basis. The trends are similar, and it can be seen that the rate of convergence of the method without online update is almost linear, as predicted by the theoretical analysis. The online update guarantees that the error on the datum is always below a given threshold and allows a significant improvement in terms of accuracy (almost one order of magnitude in certain cases). On the right panel the computational costs are shown: the number of Laplace-Beltrami basis functions (on the $\mathrm{x}$ axis) is plotted against the number of online added basis functions (on the y axis), for different tolerances, namely $\{0.01,0.005,0.001\}$ in $L_{2}(\Gamma)$ representation of the datum. The total cost in terms of number of problems $\mathcal{P}_{2}$ solved is the sum of the two.

\subsection{Two elastic cylinders in a Stokes flow}

In the last testcase, a linear elasticity problem $\left(\mathcal{P}_{1}\right)$ on two cylinders is coupled with a steady Stokes flow $\left(\mathcal{P}_{2}\right)$. This is an example of a system in which $\mathcal{P}_{1}$ is defined on a domain which is the union of non-connected sets. Moreover, both the main and the external problems have vector unknowns, and the external problem is a saddle point problem. This test is used to assess the speed-up performances of the method.

The geometry is depicted in Fig. 4. The domain $\Omega_{1}$ is composed of two cylinders attached to a basement and their displacement $\boldsymbol{\eta}$ is given by the linear elasticity equations:

$$
\mathcal{P}_{1} \begin{cases}\rho^{s} \frac{\partial^{2} \eta}{\partial t^{2}}-\operatorname{div}\left(\boldsymbol{\sigma}^{s}\right)=0 & \text { on } \Omega_{1} \\ \boldsymbol{\sigma}^{s}=\frac{E \nu}{(1+\nu)(1-2 \nu)} \operatorname{div}(\boldsymbol{\eta}) \mathbf{I}+\frac{E}{2(1+\nu)}\left(\nabla \boldsymbol{\eta}+\nabla \boldsymbol{\eta}^{T}\right) & \\ \boldsymbol{\eta}=0 & \text { on } \Gamma_{B} \\ \boldsymbol{\sigma}^{s} \boldsymbol{n}^{s}=-\boldsymbol{\sigma}^{f} \boldsymbol{n}^{f} & \text { on } \Gamma,\end{cases}
$$

where $\Gamma_{B}$, the boundary of the basement, and $\Gamma$, the union of the surfaces of the two cylinders, are such that $\bar{\Gamma}_{B} \cup \bar{\Gamma}=\partial \Omega_{1}$. The two tensors $\boldsymbol{\sigma}^{s}$ and $\boldsymbol{\sigma}^{f}$ are the stress tensors of the structure and of the fluid, respectively. We also assume that the coupling conditions between the fluid and the structure are assigned only at the cylinders boundary $\Gamma$ which, unlike the previous test cases, is not a connected set. The outward normal, with respect to $\Omega_{1}$, is denoted by $\boldsymbol{n}^{s}$ while $\boldsymbol{n}^{f}=-\boldsymbol{n}^{s}$ is outward with respect to the fluid domain $\Omega_{2}$. The physical parameter, $\rho^{s}, E$ and $\nu$ represent the density of the solid, the young modulus and the poisson ratio, respectively. For this test we have used $\rho_{s}=1, E=1 e 4, \nu=0.48$. The 

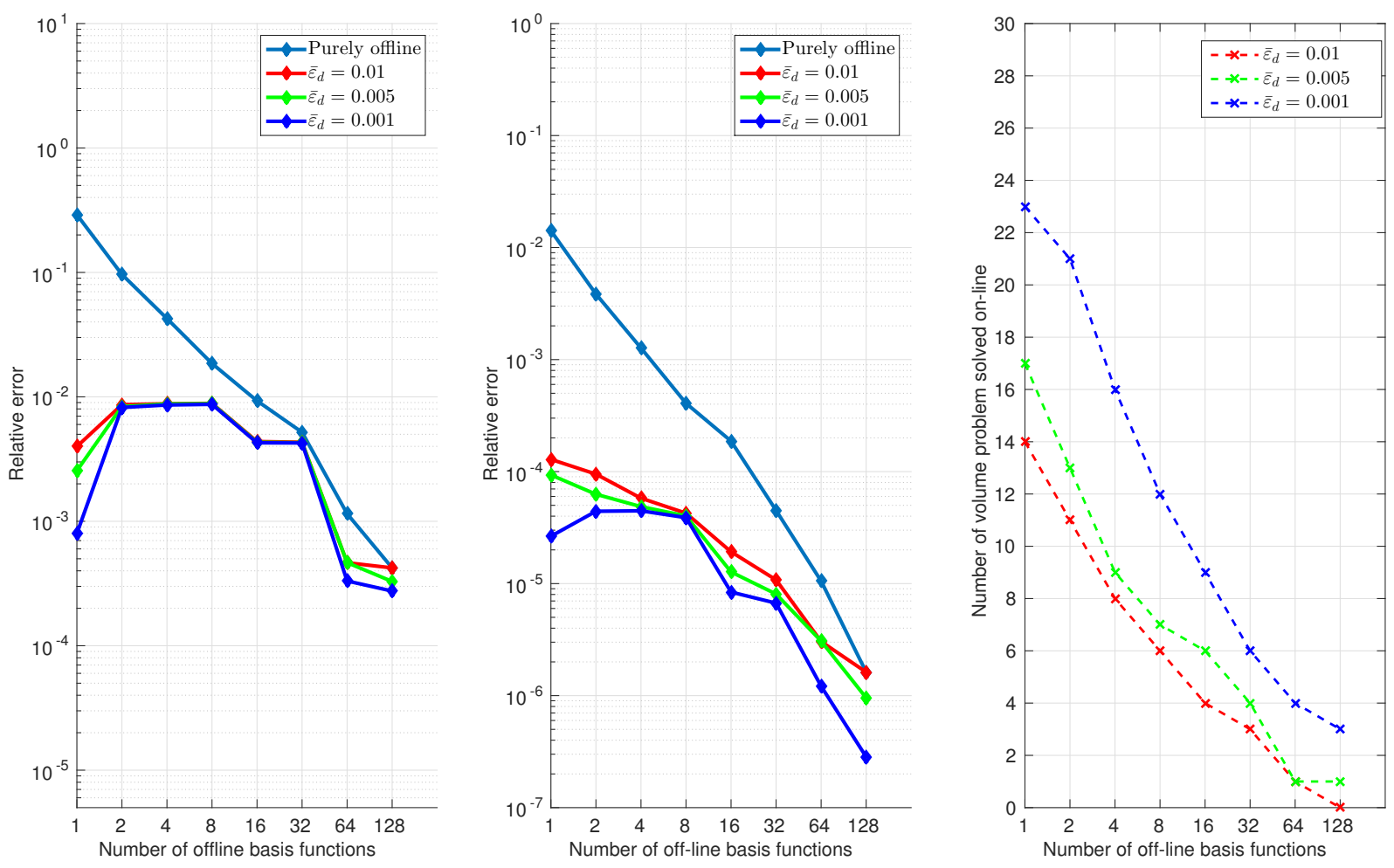

Fig. 3: In the first two panels (left and center) the error of the reduced approach with respect to the corresponding finite element simulation is computed. In the left panel the relative error is computed with respect to $\left.p_{2}\right|_{\Gamma}$ and it is measured with the $L^{2}\left((0, T) ; L^{2}(\Gamma)\right)$-norm, while in the center panel the relative error on quantity $p_{1}$ is measured with an $L^{2}\left((0, T) ; L^{2}\left(\Omega_{1}\right)\right)$ norm . The errors obtained with different approaches are compared for the offline method and for three different values of the tolerance parameter $\bar{\varepsilon}_{d}:\{0.01,0.005,0.001\}$. On the $x$-axis the number of offline basis function is reported, while on the third panel the number of basis functions that were added during the online phase is reported as a function of the offline basis dimension 


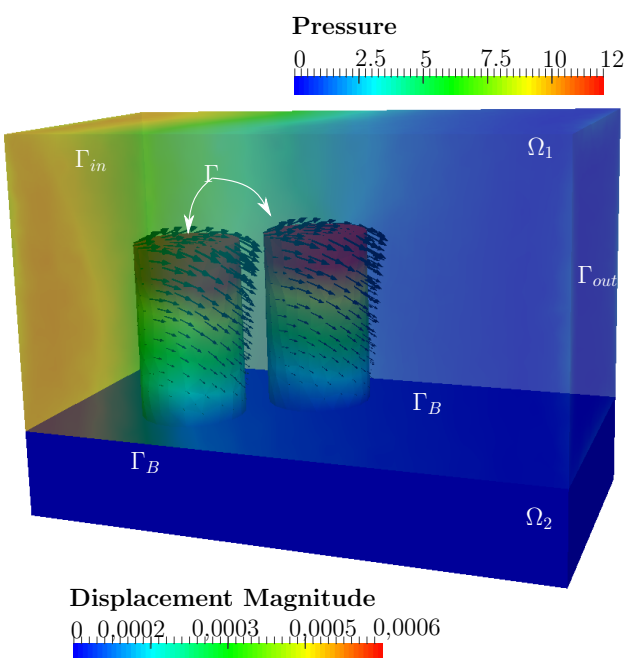

Fig. 4: Solution of the complete problem at time $t=0.00325102$. The color scale on the cylinders and on the basement represent the displacement, while the fluid domain is colored by the value of the pressure and transparent.

fluid is described by the steady Stokes equations:

$$
\mathcal{P}_{2} \begin{cases}\operatorname{div}\left(\boldsymbol{\sigma}^{f}\right)=0 & \Omega_{2} \\ \operatorname{div} \boldsymbol{u}=0 & \Omega_{2} \\ \boldsymbol{\sigma}^{f} \boldsymbol{n}^{f}=0 & \Gamma_{\text {out }} \\ \boldsymbol{\sigma}^{f} \boldsymbol{n}^{f}=-p_{\text {in }}(t) \boldsymbol{n}^{f} & \Gamma_{\text {in }} \\ \boldsymbol{\sigma}^{f}=\nu^{f}\left(\nabla \boldsymbol{u}+\nabla \boldsymbol{u}^{T}\right)-p \mathbf{I} & \\ \boldsymbol{u}=0 & \Gamma_{D} \\ \boldsymbol{u}=\dot{\boldsymbol{\eta}} & \Gamma,\end{cases}
$$

where $\boldsymbol{u}$ is the fluid velocity and $p$ the pressure. The surface $\Gamma_{i n}=\partial \Omega_{2} \cap\{x=0\}$ represents the inlet of the system. On the other side, $\Gamma_{\text {out }}=\partial \Omega_{2} \cap\{x=1\}$ is the outlet of the domain. On $\Gamma_{D}=\partial \Omega_{2} \backslash\left(\Gamma_{\text {in }} \cup \Gamma_{\text {out }} \cup \Gamma\right)$ no slip boundary conditions are enforced. The parameter $\nu^{f}$ represents the kinematic viscosity and it was set to 0.035 .

The inlet pressure is prescribed by

$$
p_{\text {in }}(t)= \begin{cases}10 & t<0.05 \\ 10\left(1-\frac{t-0.05}{0.075-0.05}\right) & 0.05<t<0.075 \\ 0 & t>0.075\end{cases}
$$

An implicit Euler scheme has been used for the time discretization of the 

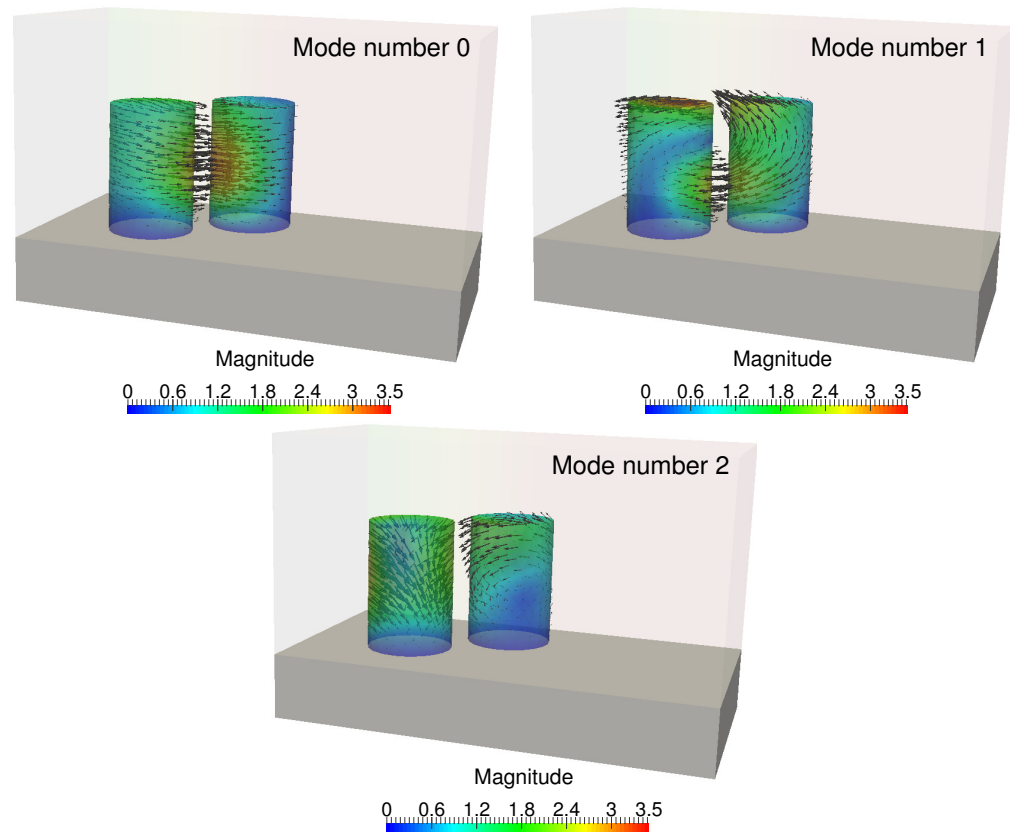

Fig. 5: First three eigen-functions of the Poincaré-Steklov operator for the Stokes problem. The reconstruction is based on 50 Laplace-Beltrami eigenfunctions.

structural problem, with a time step $\delta t=1.806125 \cdot 10^{-4}$ and P1 finite elements are used for space discretization. For the Stokes problem, we use P1-P1 stabilized finite elements. A snapshot of the reference solution obtained through this discretization is reported in Fig. 4. For what concerns space discretization we have used 20920 tetrahedra for $\Omega_{1}$ and 47735 for $\Omega_{2}$. The number of nodes at the interface is 663, which means 1989 degrees of freedom for the displacement. The final time of the simulation was $T=0.2$ for a total number of time steps equal to: 1108.

The coupling is imposed through a Dirichlet to Neumann map, that, as investigated in section 4, is featured by a slower convergence rate. The LaplaceBeltrami eigenfunctions used to sample the input space are, in this case, the solution of a vector eigenvalue problem on the cylinders surface. As a postprocessing, following Section 3.3, we can approximate the Steklov eigenfunctions and the result is shown in Figure 5. In this case, the Poincaré-Steklov eigenfunctions are non-zero on the whole interface, even though it is the union of non-connected sets. The action of the Poincaré-Steklov operator couples the movement of the two cylinders. Moreover, for the present case, the physical dissipation of the elastic cylinders is purely induced by the coupling with the external Stokes flow and hence the dissipation is related to the Steklov eigenvalues. 

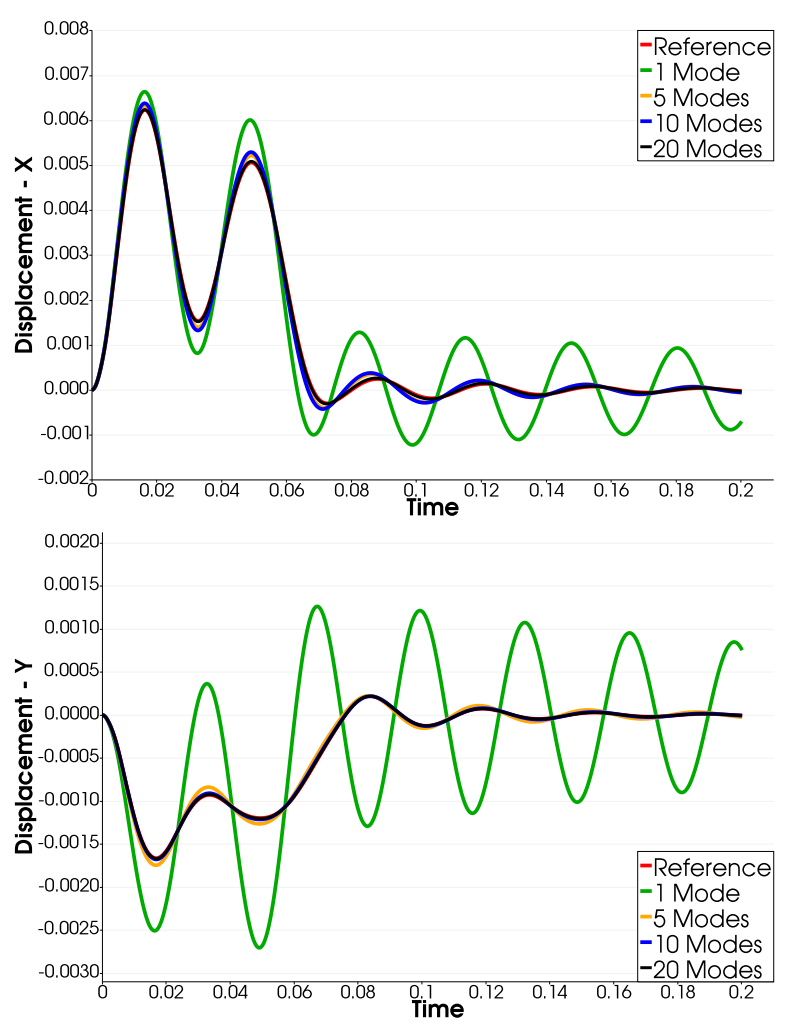

Fig. 6: The displacement of the center point on the top of the first cylinder: $x$-component on the left and $y$-component on the right. The reduced approach is compared with the reference, finite-element, solution by varying the number of modes in $\{1,5,10,20\}$.

In order to verify the quality of the reduced solution, we have reported the displacement of the center of the cylinders in Figure 6. We can see that few modal functions are enough to reproduce the correct displacement, even without online update. Observe that one basis function is, however, not sufficient to represent the system dissipation, and the oscillation of the cylinders tend to be largely under-damped.

In order to analyse in detail the computational costs of the method, different parameters are measured:

- $\tau_{\mathcal{P}_{2}}$ is the averaged time needed for one fixed point iteration for the Stokes problem;

- $\tau_{\mathcal{P}_{2}}^{*}$ takes into account also the offline costs, i.e. $\tau_{\mathcal{P}_{2}}^{*}=\tau_{\mathcal{P}_{2}}+\delta$ where $\delta$ is the amortised cost of the offline phase computed as the ratio between the total cost of the offline phase and the number of online evaluations of the reduced representation of the operator; 


\begin{tabular}{lrrrrrrrr}
\hline & $\tau_{\mathcal{P}_{2}}[s]$ & $\tau_{\mathcal{P}_{2}}^{*}[s]$ & $\boldsymbol{\zeta}_{S}$ & $\tau_{\mathcal{P}_{1}}[s]$ & $N_{d d}[-]$ & $\zeta_{d d}$ & $\tau_{\text {it }}[s]$ & $\zeta$ \\
\hline Reference & 1.06204 & 1.06425 & 1.0 & 0.640 & 6.06 & 1.00 & 10.309 & 1.00 \\
\hline$N_{\ell}=1$ & 0.00105 & 0.01181 & 1011.5 & 0.609 & 2.96 & 2.05 & 1.805 & 5.71 \\
$N_{\ell}=10$ & 0.00136 & 0.01527 & 780.9 & 0.625 & 3.43 & 1.77 & 2.145 & 4.81 \\
$N_{\ell}=20$ & 0.00173 & 0.02764 & 613.9 & 0.617 & 3.52 & 1.72 & 2.176 & 4.74 \\
$N_{\ell}=40$ & 0.00233 & 0.03872 & 455.8 & 0.629 & 3.53 & 1.72 & 2.231 & 4.62 \\
\hline
\end{tabular}

Tab. 2: Perfomance of the method for different number of basis functions.

- $\zeta_{S}$ is the ratio between $\tau_{\mathcal{P}_{2}}$ of the reference method and $\tau_{\mathcal{P}_{2}}$ of the reduced method;

- $\tau_{\mathcal{P}_{1}}$ is the averaged time needed for one fixed point iteration for the structure problem;

- $N_{d d}$ is the averaged number of domain decomposition iterations per time step;

- $\zeta_{d d}$ is the ratio between the $N_{d d}$ of the reference and the reduced method;

- $\tau_{i t}$ is the average time needed for an entire time step;

- $\zeta$ is the average speed-up per time step, i.e. the ratio of the $\tau_{i t}$ between reference and reduced methods.

The results for this test case have been obtained using GMRES preconditioned by a Restricted Additive Schwarz method, implemented in the PETSc library (PCASM), both for $\mathcal{P}_{1}$ and $\mathcal{P}_{2}$. The performances are summarized in Table 2. Observe that the reduction of the Stokes problem has been quite effective and it is between two and three orders of magnitude: the time required for the Stokes problem is negligible compared to the time needed for $\mathcal{P}_{1}$. By comparing the first two columns we also observe that, in this example, the offline costs are well amortised during the offline phase and that their impact is negligible compared to the reference cost. It is remarkable that, for this problem, the Reduced Order Model has also a regularising effect on the Domain Decomposition, so that the average number of iterations to achieve convergence is decreased with respect to the full-order method. This element contributed to the overall speed up, that, on one single simulation, results of about 4.5 (see the last two columns of the table), offline costs included.

\section{Conclusions and perspectives}

A Reduced-Order Modeling method has been presented to approximate the action of the Poincaré-Steklov operator for systems in which a non-linear complex model of interest $\left(\mathcal{P}_{1}\right)$ is coupled to an external problem $\left(\mathcal{P}_{2}\right)$. The approach aims at reducing the problem $\mathcal{P}_{2}$ whose solution is not necessarily of interest. It 
relies on an offline stage in which a sampling of possible inputs at the interface is done by means of the eigenfunctions of the Laplace-Beltrami operator. The main advantage of this choice is twofold: first, the eigenfunctions are obtained by a sparse eigenvalue problem defined at the interface only and enjoy desirable properties of approximation; second, this allows to make the offline phase independent of $\mathcal{P}_{1}$ and the coupling. The problem $\mathcal{P}_{2}$ is solved and the images of the Poincaré-Steklov operator are stored. An online update has been presented to make the method more robust to off-database cases. The numerical tests proposed, in 3D, confirmed that the method is simple and effective in a large variety of cases, being able to make the costs associated to the solution of $\mathcal{P}_{2}$ negligible compared to the costs associated to the solution of $\mathcal{P}_{1}$. Several perspectives arise, concerning the reduction of multiple external problems and networks of systems in interaction, the ability to deal with parametrized problems and the set-up of a more general approach to reduce non-linear external problems.

\section{Aknowledgements}

This research was made possible by a Marie Curie grant from the European Commission in the framework of the REVAMMAD ITN (Initial Training Research network), Project number 316990.

\section{References}

[1] Quarteroni A, Valli A. Domain Decomposition methods for partial differential equations. Oxford University Press, 1999.

[2] Agoshkov V, Lebedev V. Poincaré-steklov's operators and the methods of partition of the domain in variational problems. Computational Processes and Systems 1985; 2:173-227.

[3] Deparis S, Discacciati M, Fourestey G, Quarteroni A. Fluid-structure algorithms based on Steklov-Poincaré operators. Computer Methods in Applied Mechanics and Engineering 2006; 195(41):5797-5812.

[4] Constantine PG, Phipps ET, Wildey TM. Efficient uncertainty propagation for network multiphysics systems. International Journal for Numerical Methods in Engineering 2014; 99(3):183-202.

[5] Paz RR, Storti MA. An interface strip preconditioner for domain decomposition methods: application to hydrology. International journal for numerical methods in engineering 2005; 62(13):1873-1894.

[6] Gendre L, Allix O, Gosselet P. A two-scale approximation of the Schur complement and its use for non-intrusive coupling. International Journal for Numerical Methods in Engineering 2011; 87(9):889-905. 
[7] Natarajan R. Domain decomposition using spectral expansions of Steklov-Poincaré operators. SIAM Journal on Scientific Computing 1995; 16(2):470-495.

[8] Natarajan R. Domain decomposition using spectral expansions of SteklovPoincaré operators ii: A matrix formulation. SIAM Journal on Scientific Computing 1997; 18(4):1187-1199.

[9] Cao L, Zhang L, Allegretto W, Lin Y. Multiscale asymptotic method for Steklov eigenvalue equations in composite media. SIAM Journal on $\mathrm{Nu}$ merical Analysis 2013; 51(1):273-296.

[10] Liew HL, Pinsky PM. Matrix-Padé via Lanczos solutions for vibrations of fluid-structure interaction. International Journal for Numerical Methods in Engineering 2010; 84(10):1183-1204.

[11] Fang Z, Nicholls DP. An operator expansions method for computing Dirichlet-Neumann operators in linear elastodynamics. Journal of Computational Physics 2014; 272:266-278.

[12] Bélanger-Rioux R, Demanet L. Compressed absorbing boundary conditions via matrix probing. SIAM Journal on Numerical Analysis 2015; $\mathbf{5 3}(5): 2441-2471$.

[13] Huynh DBP, Knezevic DJ, Patera AT. A static condensation reduced basis element method: approximation and a posteriori error estimation. ESAIM: Mathematical Modelling and Numerical Analysis 2013; 47(1):213-251.

[14] Eftang JL, Patera AT. A port-reduced static condensation reduced basis element method for large component-synthesized structures: approximation and a posteriori error estimation. Advanced Modeling and Simulation in Engineering Sciences 2014; 1(1):1.

[15] Martini I, Rozza G, Haasdonk B. Reduced basis approximation and aposteriori error estimation for the coupled Stokes-Darcy system. Advances in Computational Mathematics 2015; 41(5):1131-1157.

[16] Iapichino L, Quarteroni A, Rozza G. Reduced basis method and domain decomposition for elliptic problems in networks and complex parametrized geometries. Computers $\&$ Mathematics with Applications 2016; 71(1):408430.

[17] Quarteroni A, Rozza G. Reduced Order Methods for Modeling and Computational Reduction, vol. 9. Springer, 2014.

[18] Peherstorfer B, Willcox K. Online adaptive model reduction for nonlinear systems via low-rank updates. SIAM Journal on Scientific Computing 2015; 37(4):A2123-A2150. 
[19] Amsallem D, Zahr MJ, Washabaugh K. Fast local reduced basis updates for the efficient reduction of nonlinear systems with hyper-reduction. Advances in Computational Mathematics 2015; 41(5):1187-1230.

[20] Toselli A, Widlund O. Domain decomposition methods: algorithms and theory, vol. 34. Springer, 2005.

[21] Smith B, Bjorstad P, Gropp W. Domain decomposition: parallel multilevel methods for elliptic partial differential equations. Cambridge university press, 2004.

[22] Marini LD, Quarteroni A. A relaxation procedure for domain decomposition methods using finite elements. Numerische Mathematik 1989; 55(5):575-598.

[23] Douglas J, Huang CS. An accelerated domain decomposition procedure based on Robin transmission conditions. BIT Numerical Mathematics 1997; $37(3): 678-686$.

[24] Negri F, Manzoni A, Amsallem D. Efficient model reduction of parametrized systems by matrix discrete empirical interpolation. Journal of Computational Physics 2015; 303:431-454.

[25] Khoromskij BN, Wittum G. Numerical solution of elliptic differential equations by reduction to the interface, vol. 36. Springer Science \& Business Media, 2012.

[26] Canuto C, Hussaini M, Quarteroni A, Zang T. Spectral Methods: Fundamentals in Single Domains. Scientific Computation, Springer Berlin Heidelberg, 2007.

[27] Aletti M, Perotto S, Veneziani A. Educated bases for the HiMod reduction of advection-diffusion-reaction problems with general boundary conditions. Technical Report, MOX Report 2015.

[28] Salsa S. Partial differential equations in action: from modelling to theory, vol. 86. Springer, 2015.

[29] Bergh J, Lofstrom J. Interpolation spaces: an introduction, vol. 223. Springer Science \& Business Media, 2012.

[30] Gholami A, Malhotra D, Sundar H, Biros G. FFT, FMM, or Multigrid? a comparative study of state-of-the-art Poisson solvers in the unit cube. SIAM Journal on Scientific Computing 2016; 38.

[31] Discacciati M, Quarteroni A. Numerical Mathematics and Advanced Applications: Proceedings of ENUMATH 2001 the 4th European Conference on Numerical Mathematics and Advanced Applications Ischia, July 2001, chap. Analysis of a domain decomposition method for the coupling of Stokes and Darcy equations. Springer Milan: Milano, 2003; 3-20, doi: 10.1007/978-88-470-2089-4_1. 
[32] Nobile F, Vergara C. An effective fluid-structure interaction formulation for vascular dynamics by generalized robin conditions. SIAM Journal on Scientific Computing 2008; 30(2):731-763, doi:10.1137/060678439.

\section{Appendix A}

In this part, a sufficient condition for the eigenfunctions of the Poincaré-Steklov operator to coincide with the eigenfunctions of the Laplace-Beltrami operator on the interface is given.

Proposition 2. Let $\Omega_{2}=\mathcal{M}_{1} \otimes \ldots \otimes \mathcal{M}_{d} \subseteq \mathbb{R}^{d}$ be the domain for $\mathcal{P}_{2}$. It is expressed as the cartesian product of $1 D$ manifolds, and it admits a generalised orthonormal set of coordinates such that the Laplace equation is separable. Moreover $\Gamma$ is a codimension-1 submanifold, which is defined by $x_{1}=0$. Let $\mathcal{P}_{2}$ be the Laplace equation defined on $\Omega_{2}$. Then the eigenfunctions of the Poincaré-Steklov operator coincide with the eigenfunctions of the Laplace-Beltrami operator defined on $\Gamma$.

Proof. The Laplace equation written for a generic orthonormal curvilinear system reads:

$$
\Delta u_{2}=\sum_{j=1}^{d} \frac{1}{h_{j}} \partial_{x_{j}}\left(g_{j} \partial_{x_{j}} u_{2}\right)=0,
$$

where $h_{j}, g_{j}$ contains the metric factors and are functions of the coordinates. The Steklov eigenvalue problem reads:

$$
\begin{array}{r}
-\Delta u_{2}=0, \text { on } \Omega_{2} \\
\partial_{n} u_{2}+\alpha u_{2}=\lambda u_{2}, \text { on } \Gamma \\
u_{2}=0, \quad \text { on } \partial \Omega_{2} / \Gamma .
\end{array}
$$

By hypothesis $u_{2}$ admits a separable representation, i.e.

$$
u_{2}=\prod_{j=1}^{d} \hat{u}_{j}\left(x_{j}\right)
$$

This expression is plugged into the eigenvalue equation leading to:

$$
\begin{array}{r}
-\sum_{j=1}^{d}\left(\prod_{k \neq j}^{d} \hat{u}_{k}\right) \frac{1}{h_{j}} \partial_{x_{j}}\left(g_{j} \partial_{x_{j}} \hat{u}_{j}\right)=0, \text { on } \Omega_{2} \\
\frac{1}{h_{1}} \partial_{x_{1}} \hat{u}_{1}\left(\prod_{k \neq 1}^{d} \hat{u}_{k}\right)+(\alpha-\lambda)\left(\prod_{k=1}^{d} \hat{u}_{k}\right)=0, \text { on } \Gamma \\
\left(\prod_{k=1}^{d} \hat{u}_{k}\right)=0, \quad \text { on } \partial \Omega_{2} / \Gamma .
\end{array}
$$


The system is equivalent to the following:

$$
\begin{array}{r}
-\sum_{j=1}^{d} \frac{1}{\hat{u}_{j}} \frac{1}{h_{j}} \partial_{x_{j}}\left(g_{j} \partial_{x_{j}} \hat{u}_{j}\right)=0, \text { on } \Omega_{2} \\
\frac{1}{h_{1}} \partial_{x_{1}} \hat{u}_{1}+(\alpha-\lambda) \hat{u}_{1}=0, \text { on } \Gamma \\
\left(\prod_{k=1}^{d} \hat{u}_{k}\right)=0, \quad \text { on } \partial \Omega_{2} / \Gamma .
\end{array}
$$

The first equation can be decoupled into a system of $d$ independent equations of the form:

$$
\frac{1}{h_{j}} \partial_{x_{j}}\left(g_{j} \partial_{x_{j}} \hat{u}_{j}\right)=c_{j} \hat{u}_{j} .
$$

with the closure condition:

$$
-\sum_{j}^{d} c_{j}=0
$$

On all the boundary except for $\Gamma$ there are homogeneous boundary conditions. The solution of the $d-1$ equation for the coordinates $x_{2, \ldots, d}$ is thus obtained by using $c_{j}<0$ and it is a set of harmonic oscillations in the direction $x_{j}$. As consequence $c_{1}>0$ and it holds:

$$
c_{1}=-\sum_{j=2}^{d} c_{j} .
$$

Moreover, when considering the conditions on $\Gamma$, the equation along $x_{1}$ reads:

$$
\begin{array}{r}
\frac{1}{h_{1}} \partial_{x_{1}}\left(g_{j} \partial_{x_{1}} \hat{u}_{1}\right)=c_{1} \hat{u}_{1}, \\
\frac{1}{h_{1}} \partial_{x_{1}} \hat{u}_{1}+(\alpha-\lambda) \hat{u}_{1}=0, \text { on } \Gamma \\
\hat{u}_{1}=0, x_{1}=1 .
\end{array}
$$

When solved, this equation provides a relationship between $\lambda$ and $c_{j}$. When restricted to $\Gamma$ the solution of the problem reads:

$$
\left.u_{2}\right|_{\Gamma}=\hat{u}_{1}(0)\left(\prod_{j=2}^{d} \hat{u}_{j}\left(x_{j}\right)\right)
$$

which is the set of the Laplace-Beltrami eigenfunctions on $\Gamma$ up to normalisation.

Although the hypothesis for this condition to hold true are quite restrictive, they are common to a broad class of different problems and geometrical settings (think for instance to boxes, concentrical cylinders). 